\title{
EVERYWHERE DENSE SUBGROUPS OF LIE GROUPS
}

\author{
P. A. SMITH
}

A recent note by Montgomery and Zippin ${ }^{1}$ leads one to speculate concerning the nature of everywhere dense proper subgroups of continuous groups. Such subgroups can easily be constructed. Suppose for example that $G$ is a non-countable continuous group which admits a countable subset $G_{0}$ filling it densely. The group generated by $G_{0}$ is everywhere dense in $G$ but is not identical with $G$. In the case of Lie groups, it is easy to see that an abelian $G$ admits non-countable subgroups of the sort in question; whether or not a non-abelian $G$ does so, appears to be a more difficult question. We shall, however, show that if $G$ is simple, proper subgroups of $G$ cannot, so to speak, fill $G$ too densely.

Let $G$ be a simple ${ }^{2}$ Lie group of dimension $r$ with $r>1$, and let $U$ be a canonical nucleus of $G$-that is, a nucleus which can be covered by an analytic canonical coordinate system. An arbitrary point $x$ of $U$ is contained in the central of at least one closed proper Lie subgroup of $G$ with non-discrete central. In fact, through $x$ there passes a oneparameter subgroup $\gamma$; the closure of $\gamma$ is an abelian Lie subgroup and this subgroup is proper since $G$ is simple and $r>1$.

THeOREM. Let $G$ be a simple Lie group of dimension $r$ greater than one and let $\mathfrak{g}$ be a proper subgroup filling $G$ densely. There exists at least one proper closed Lie subgroup $H$ of $G$ such that those left- (right-) cosets of $H$ which fail to meet $\mathrm{g}$ fill $G$ densely. For $H$ one may take any closed proper Lie subgroup of $G$ whose central is non-discrete and contains an arbitrarily chosen point $p$ in $\mathfrak{g} \cap U, U$ being any given canonical nucleus of $G$.

Proof. Let $U, p, H$ be chosen and let us consider only the leftcosets of $H$. It will be sufficient to prove that there exists at least one coset, say $a H$, which fails to meet $g$. For, the cosets obtained by multiplying $a H$ on the left by arbitrary elements of $\mathfrak{g}$ fail to meet $\mathfrak{g}$ and fill $G$ densely.

Received by the editors July 15, 1941.

${ }^{1}$ Deane Montgomery and Leo Zippin, $A$ theorem on the rotation group of the 2sphere, this Bulletin, vo!. 46 (1940), pp. 520-521. Our theorem may be regarded as a generalization of the theorem of Montgomery and Zippin and the proofs of the two theorems may be regarded as being the same in principle.

${ }^{2}$ We use simple here in the sense of having a simple Lie algebra. A simple group need not be connected. 
Let us assume the contrary, namely that every coset of $H$ meets $\mathfrak{g}$. Let $H^{*}$ be the totality of cosets of $H$ and let the elements of $H^{*}$ be denoted by $e^{*}=H, a^{*}=a H, \cdots$. Let $\sigma$ be the mapping $x \rightarrow x^{*}$ $\left(x^{*}=x H\right)$ of $G$ into $H^{*}$. Let $H^{*}$ be topologized in the usual way by taking as open in $H^{*}$ every set of the form $\sigma A$ where $A$ is an open subset of $G$. The space $H^{*}$ is homogeneously locally euclidean.-Now let $x^{*}$ be an element of $H^{*}$ and let $x$ be a representative of the coset $x^{*}$. Then $x p x^{-1}$ (where $p$ is defined in the theorem) is independent of $x$. For if $y$ is a second representative of $x^{*}$, then $x^{-1} y \subset H$ so that $x^{-1} y p=p x^{-1} y$ since $p$ is in the central of $H$. Hence $x p x^{-1}=y p y^{-1}$. Thus the formula $\tau\left(x^{*}\right)=x p x^{-1}$ defines a mapping $\tau$ of $H^{*}$ into $G$ which, in particular, carries $e^{*}$ into $p$. Evidentally $\tau$ is continuous. In fact it is easy to see that $\tau$ is analytic relative to an arbitrarily chosen analytic canonical coordinate system $x_{1}, \cdots, x_{r}$ covering $U$, and a suitably chosen coordinate system covering a neighborhood of $e^{*}$.

The mapping $\tau$ carries $H^{*}$ into a subset of $\mathfrak{g}$. For, by our assumption on the cosets of $H$, an element $y^{*}$ of $H^{*}$ can be written in the form $y^{*}=g H$ where $g \subset g$. Hence we have $\tau\left(y^{*}\right)=g p g^{-1} \subset g$.-Moreover, any given neighborhood $V^{*}$ of $e^{*}$ contains at least one point $x^{*}$ such that $\tau\left(x^{*}\right) \neq p$. For otherwise we have $\tau(y H)=p$ for every $y$ in a certain nucleus $V$ of $G$, that is, for every $y$ in $V$ and $h$ in $H$ we have $y h p(y h)^{-1}$ $=p$ or $y p y^{-1}=p$. But then the one-parameter subgroup of $G$ determined by $p$ would be invariant, contrary to the hypothesis that $G$ is simple.

Let $W$ be a nucleus of $G$ such that $W^{-1} W W \subset U$. It follows from the last two paragraphs that there exists in $H^{*}$ a point $z^{*}$ near $e^{*}$ such that the linear segment $e^{*} z^{*}$ is carried by $\tau$ into an analytic arc contained in $\mathfrak{g} \cap W$ and consisting of more than a single point. A suitably chosen piece of this arc, when multiplied on the left by the inverse of one of its points, furnishes an analytic 1 -cell $K$ contained in $\mathfrak{g} \cap W$ and containing $e$, the identity of $G$. Starting with $K$ we shall construct a dimensionally increasing sequence of analytic continua, subsets of $\mathfrak{g}$. In what follows, let it be understood that all functions are real, singlevalued and analytic over the domains indicated.

We may suppose that $K$ is defined parametrically, say by $x_{i}=f_{i}(t)$ where $-1<t<1$ and $f(0)=e$. The set $K K$ is in $g$ and is defined by equations of the form $x_{i}=g_{i}(s, t)$ where $-1<s, t<1$. Suppose that $\operatorname{dim} K K>\operatorname{dim} K$; that is, suppose $\operatorname{dim} K K=2$. Then being an analytic locus, $K K$ contains points at which it is locally euclidean 2-dimensional. If $b$ is such a point, then $b^{-1} K K$ (a subset of $\mathfrak{g}$ ) is locally euclidean at $e$. Hence $\mathfrak{g} \cap W$ contains a 2-cell $K_{2}$ defined say by $x_{i}=h_{i}(u, v)$ where $-1<u, v<1$ and $h(0,0)=e$. We next consider the 
set $K_{2} K_{2}$ and suppose that its dimension exceeds that of $K_{2}$. On continuing in this manner, we finally obtain a $k$-cell $E$ in $\mathfrak{g} \cap W$ defined say by $x_{i}=h_{i}\left(u_{1}, \cdots, u_{k}\right)$ where $-1<u_{i}<1$ and $h(0, \cdots, 0)=e$, and such that $\operatorname{dim} E E=\operatorname{dim} E=k$. We assert that $E$ contains subsets $E^{*}$ and $F$ such that (1) $E^{*}$ and $F$ are $k$-cells; (2) $e \subset F \subset E^{*}$; (3) $F F \subset E^{*}$.

To prove this, we first note that by the theory of implicit functions, $E$ contains a $k$-dimensional sub-cell $E^{*}$ definable, after renaming the coordinates $x_{i}$ if necessary, by equations

$$
x_{i}=X_{i}\left(x_{1}, \cdots, x_{k}\right), \quad i=k+1, \cdots, r,
$$

where $\left(x_{1}, \cdots, x_{k}\right)$ ranges over the cube $C_{\delta}:-\delta<x_{i}<\delta$, and where $X_{i}(0, \cdots, 0)=e_{i}=0$. On replacing $\delta$ by a smaller number if necessary, it is easy to see that $C_{\delta}$ contains a cube $C_{\mu}:-\mu<x_{i}<\mu$ $(i=1, \cdots, h)$ such that if $F$ is the $k$-cell defined by (1) with $\left(x_{1}, \cdots, x_{k}\right)$ restricted to the cube $C_{\mu}$, and if $q$ is an arbitrary point of $F$, then $q F$, like $F$, is definable by equations of the form (1):

$$
x_{i}=X_{i}^{q}\left(x_{1}, \cdots, x_{k}\right)
$$

where $\left(x_{1}, \cdots, x_{k}\right)$ ranges over a certain open subset $A^{q}$ of $C_{\delta}$. Now $E E$ is the union of $k$-cells $q E(q \subset E)$, hence is $k$-dimensional at every point. Being an analytic locus, the points $q$ at which $E E$ is locally euclidean $k$-dimensional fill it densely. Consider such a point $q$. The $k$-cells $F$ and $q F$ intersect at $q$. But since both are contained in $E E$ which is locally euclidean $k$-dimensional at $q$, they coincide identically in the neighborhood of $q$. Hence the functions $X_{i}$ and $X_{i}^{q}$ are identically equal over an open subset of $A^{q}$; hence, by the theory of analytic functions, they are equal over the whole of $A^{q}$. Hence $q F \subset E^{*}$, and this is true for a set of points $q$ filling $F$ densely. By continuity this relation holds for arbitrary $q$ in $F$. Hence $F F \subset E^{*}$, proving our assertion.

It is easy to see that on replacing $F$ by a smaller $k$-cell if necessary, we have also $F^{-1} \subset E^{*}$. In short $F$ is a $k$-dimensional local Lie subgroup of $G$; hence it is an open subset of a $k$-dimensional linear subspace $L$ of the linear space of the canonical coordinates $x_{1}, \cdots, x_{r}$. If $k<r$, there exists in $W$ an element $a$ such that the linear subspace $L^{\prime}$ determined by $F^{\prime}=a F a^{-1}$ is different from $L$; otherwise the Lie subalgebra represented by $L$ is invariant. Since $\mathfrak{g}$ is everywhere dense in $G$, we may assume, so far as the relation $L \neq L^{\prime}$ is concerned, that $a \subset g$. Then $F F^{\prime} \subset$ g. Moreover, it is evident that $\operatorname{dim} F F^{\prime}>k$. We can now repeat the construction described above starting with a suitably chosen analytic cell of dimension exceeding $k$ in $F F^{\prime}$. We obtain 
finally an analytic $r$-cell contained in $\mathfrak{g} \cap W$. Hence $\mathfrak{g}$ contains a nucleus of $G$ and hence $\mathfrak{g}=G$, a contradiction which proves the theorem. ${ }^{3}$

Columbia University

${ }^{3}$ We have proved, incidentally, that if an everywhere dense subgroup $\mathfrak{g}$ of a simple Lie group $G_{r}(r>1)$ contains an analytic arc, then $\mathfrak{g}=G$.

\section{VECTOR SPACES OVER RINGS}

\section{J. EVERETT ${ }^{1}$}

1. Introduction. Let $\mathfrak{M}=u_{1} K+\cdots+u_{m} K$ be a vector space (linear form modul [5, p. 111]) over a ring $K=\{0, \alpha, \beta, \ldots ; \epsilon$ unit element $\}$. By a submodul $\mathfrak{N} \leqq \mathfrak{M}$ is meant an "admissible" submodul: $\mathfrak{N} \leqq \leqq$. Elements $v_{1}, \cdots, v_{n}$ of a submodul $\mathfrak{N}$ form a basis for $\mathfrak{N}$ (notation: $\mathfrak{N}=v_{1} K+\cdots+v_{n} K$ ) in case $\sum v_{i} \alpha_{i}=0$ implies $\alpha_{i}=0$, $i=1, \cdots, n$, and if every element of $\mathfrak{N}$ is expressible in the form $\sum v_{i} \alpha_{i}, \alpha_{i} \in K$. The equivalent formulations of the ascending chain condition for submoduls of a vector space, and for right ideals of a ring will be used without further comment $[5, \S \S 80,97]$.

2. Basis number, linear transformations. We remark that the following holds.

(A) The ascending chain condition is satisfied by the submoduls of a vector space $\mathfrak{M}$ over $K$ if and only if it is satisfied by the right ideals of $K$.

An infinite chain of right ideals $\mathfrak{r}_{1}<\mathfrak{r}_{2}<\cdots$ in $K$ yields an infinite chain of submoduls $u_{1} \mathfrak{r}_{1}<u_{1} \mathfrak{r}_{2}<\ldots$ in $\mathfrak{M}$. The other implication is proved in $[5$, p. 87$]$.

[By using a lemma due to N. Jacobson (Theory of Rings, in publication) Theorem (A) and the corresponding theorem for descending chain condition are easily proved in a unified manner.]

Linear transformations of $\mathfrak{M}$ on $\mathfrak{M}$ are given by $u_{j} \rightarrow u_{j}^{\prime}=\sum u_{i} \alpha_{i j}$. Write $\left(u_{1}^{\prime}, \cdots, u_{m}^{\prime}\right)=\left(u_{1}, \cdots, u_{m}\right) A, A=\left(\alpha_{i j}\right)$. Under $u_{j} \rightarrow u_{j}^{\prime}$, let $\mathfrak{M}_{0} \rightarrow 0$. Thus $\mathfrak{M} / \mathfrak{M}_{0} \cong \mathfrak{M} A \leqq \mathfrak{M}$. Clearly $\mathfrak{M}_{0}=0$ if and only if $A v=0$ implies $v=0, v$ an $m \times 1$ matrix over $K$, and $\mathfrak{M} A=\mathfrak{M}$ if and only if there exists an $m \times m$ matrix $R$ with $A R=I$, the identity matrix.

Possibilities (i) $\mathfrak{M}_{0}=0$ and $\mathfrak{M} A=\mathfrak{M}$; (ii) $\mathfrak{M}_{0}>0$ and $\mathfrak{M} A<\mathfrak{M}$; (iii) $\mathfrak{M}_{0}=0$ and $\mathfrak{M} A<\mathfrak{M}$ are familiar. The possibility of (iv) $\mathfrak{M}_{0}>0$

Presented to the Society, September 5, 1941; received by the editors May 27, 1941.

1 The results presented here were obtained while the author was Sterling Research Fellow in mathematics, Yale University, 1940-1941. Thanks are due to Professors Oystein Ore, R. P. Dilworth, and the referee for helpful suggestions. 